\title{
Les réacteurs catalytiques gaz/liquide/solide Problèmes à résoudre et méthodologie suivie
}

\author{
Gas/liquid/solid catalytic reactors \\ Problems to solve and methodoly employed \\ par C. Boyer \\ Institut Français du Pétrole \\ Centre d'Etudes et de Développement Industriel de Solaize \\ Division du Génie des Procédés
}

This paper proposes a review of hydrodynamics problems encountered in catalytic reactor characterized by a fixed catalyst bed and crossed by gas/liquid or liquid flows. These reactors are typical reactors of petrochemical or refinery processes. These hydrodynamic problems are specifically studied at the French Institute of Petroleum center of Solaize (IFP/Solaize). Once background of these studies presented, the different instrumentation techniques are described by emphasizing on development needs. Finally, at the end of the paper, a special attention is paid to instrumentation and modeling needs concerning gasliquid flow across a fixed bed of solid particle.

\section{I $\mathbf{I N T R O D U C T I O N}$}

De nombreux procédés de raffinage et de pétrochimie sont basés sur des réactions mettant en jeu une phase liquide et une phase gaz et nécessitant l'utilisation de catalyseur. La phase active étant fixée sur des particules solides, le procédé fait intervenir un écoulement liquide/gaz et un lit de particules solides.

Dans les réacteurs catalytiques qui sont décrits dans cet article, le lit de particules solides est maintenu immobile et est traversé par l'écoulement gaz/liquide. On appelle ces réacteurs des "réacteurs à lit fixe". Ils sont notamment utilisés dans les unités d'hydrogénation sélective, d'hydrodésulfuration..

Le rendement de la réaction est bien sûr dépendant des caractéristiques hydrodynamiques de l'écoulement et en particulier de la composition du mélange liquide/gaz en contact avec le catalyseur. Par exemple, la présence de zones du lit balayées par du gaz seul peut avoir des conséquences graves : pertes de sélectivité, désactivation du catalyseur, montée en température... De nombreuses études s'attachent à analyser les problèmes d'hydrodynamique de ces réacteurs et à comprendre en particulier l'origine des défauts de distribution des phases liquide et gaz suivant différentes sections du réacteur chimique. Le but final de ces études est de proposer le dimensionnement d'internes permettant de résoudre ces problèmes de mauvaise distribution et d'optimiser ainsi le contact entre la phase gaz, la phase liquide et le catalyseur.

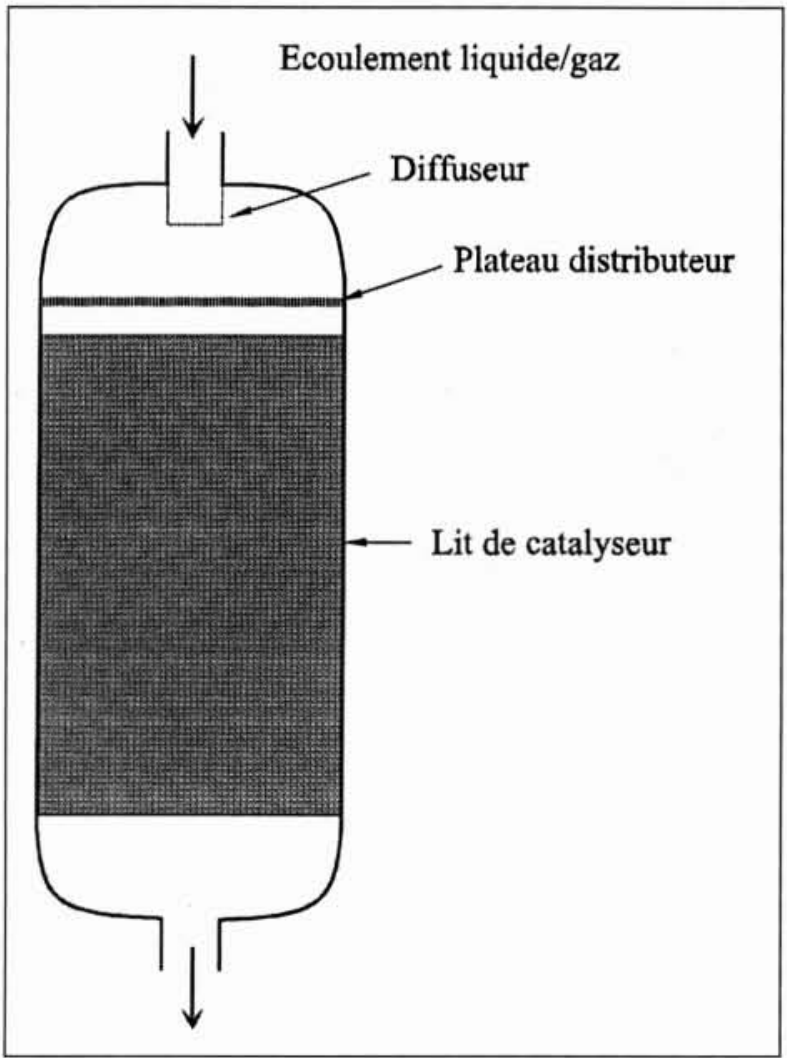

1. Schéma général d'un réacteur gaz/liquide/solide de type "lit fixe". 


\section{II $\square$ CONTEXTE DES ÉTUDES}

Comme le montre la figure 1 , les réacteurs étudiés sont composés de manière générale de trois parties :

- l'entrée du réacteur : elle comprend un système de distribution composé d'un plateau distributeur associé ou non à un diffuseur.

- le lit de particules solides : la forme de ces particules peut être sphérique ou cylindrique et leur taille varie de quelques dixièmes de $\mathrm{mm}$ à quelques $\mathrm{mm}$. Ces "grains" sont poreux pour augmenter au maximum la surface de contact entre les réactifs et le catalyseur. Par simplification, le terme de "catalyseur" est souvent associé aux particules qui lui servent de support et le lit de particules solides est ainsi appelé "lit de catalyseur".

- la sortie du réacteur.

La configuration retenue pour la figure 1 correspond à un écoulement de type descendant mais ces réacteurs peuvent être utilisés indifféremment en écoulement descendant ou ascendant. La gamme de taux de vide dans l'écoulement liquide/gaz amont peut varier de 0 à 1 suivant le procédé étudié. De même les conditions de débit, pression et température sont variables d'un procédé à l'autre.

La section à l'amont du lit a pour rôle d'optimiser la distribution des phases liquide et gaz à l'entrée du réacteur. La forme et les caractéristiques du plateau distributeur et du diffuseur font appel à différentes technologies qui ne seront pas données ici pour des raisons de confidentialité. Les caractéristiques du lit peuvent être aussi variables : densité du chargement, granulométrie des particules solides... De même, le lit de catalyseur peut être scindé en plusieurs parties avec un dispositif de redistribution intermédiaire entre chacune des parties.

Au centre de Solaize, les études concernant ces réacteurs liquide/gaz/solide sont réalisées sur des unités représentatives des réacteurs industriels à petite échelle. La division du Génie des Procédés de l'IFP dispose à cet effet de plusieurs maquettes dont le diamètre varie de 50 à $1600 \mathrm{~mm}$. Ces unités sont destinées uniquement à l'étude des problèmes d'hydrodynamique, la réaction chimique n'étant pas prise en compte. Les fluides utilisés sont généralement de l'heptane pour la phase liquide et de l'azote pour la phase gaz. La température de fonctionnement est proche de la température ambiante et la pression varie entre la pression atmosphérique et 10 bar en entrée. Les débits de liquide et de gaz sont déterminés de façon à être représentatifs des vitesses superficielles utilisées dans les réacteurs industriels.

\section{III — MÉTHODOLOGIE EXPÉRIMENTALE}

Les méthodes d'investigation expérimentale utilisées pour caractériser et analyser les écoulements diphasiques dans les réacteurs gaz/liquide/solide peuvent être classées en deux groupes suivant le caractère global ou local des paramètres mesurés.

\subsection{Mesures globales}

\subsubsection{Tomographie gamma}

Pour connaître la répartition spatiale des phases liquide et gaz suivant une section du réacteur, les seules méthodes globales existantes sont basées sur le principe de la tomographie. Parmi les technologies testées pour reconstruire une image tomographique d'une section de conduite, les mesures électriques n'offrent qu'une très mauvaise résolution spatiale et ce d'autant plus que l'on s'éloigne des parois. En effet la tomographie à impédance permet surtout de détecter des variations rapides de la composition de l'écoulement mais reste très peu adaptée pour détecter avec précision la cartographie de la phase gazeuse dans l'écoulement. Les méthodes plus marginales basées sur l'utilisation de techniques ultrasonores ou micro-ondes n'ayant pas pour l'instant fait l'objet d'un développement suffisant pour être validées, les seules techniques utilisables restent les mesures d'atténuation photoniques.

Compte tenu des épaisseurs à traverser, la tomographie par rayons $\mathrm{X}$ impose l'utilisation de photons d'énergie très élevée dont la mise en œuvre est très difficilement concevable dans un environnement pour lequel les normes d'antidéflagrance (ADF) doivent être respectées. L'utilisation de rayonnement gamma a donc été préféré pour effectuer les mesures d'atténuation. Le dispositif de mesure est décrit à la figure 2 .

Dans sa configuration actuelle, le dispositif utilisé est composé d'une source de Cesium 137 d'activité $300 \mathrm{mCi}$ et d'énergie $660 \mathrm{keV}$ et d'un détecteur de type scintillateur NaI. Le couple émetteur-détecteur est disposé sur un support tournant sur lequel il peut effectuer des mouvements de translation et de rotation autour de la conduite. Le diamètre de collimation étant de $1 \mathrm{~cm}$, les acquisitions sont effectuées tous les $\mathrm{cm}$, ce qui permet de reconstruire une image de $64 \times 64$ pixels pour la section du réacteur de $600 \mathrm{~mm}$ de diamètre.

\section{Limitations}

Le problème majeur est évidemment le temps de mesure nécessaire pour scruter l'ensemble de la section. Compte tenu de l'activité de la source et de l'épaisseur traversée il faut en effet $10 \mathrm{~s}$ par point de mesure pour obtenir une précision statistique convenable sur le comptage mesuré. Le temps total de mesure est donc excessif pour permettre des mesures valides en dynamique pour lesquelles l'hypothèse de stationnarité de l'écoulement doit rester vérifiée. Aussi, l'amélioration du dispositif actuel est-elle à l'étude pour diminuer ce temps de comptage en utilisant une géométrie de faisceau différente, un plus grand nombre de détecteurs..

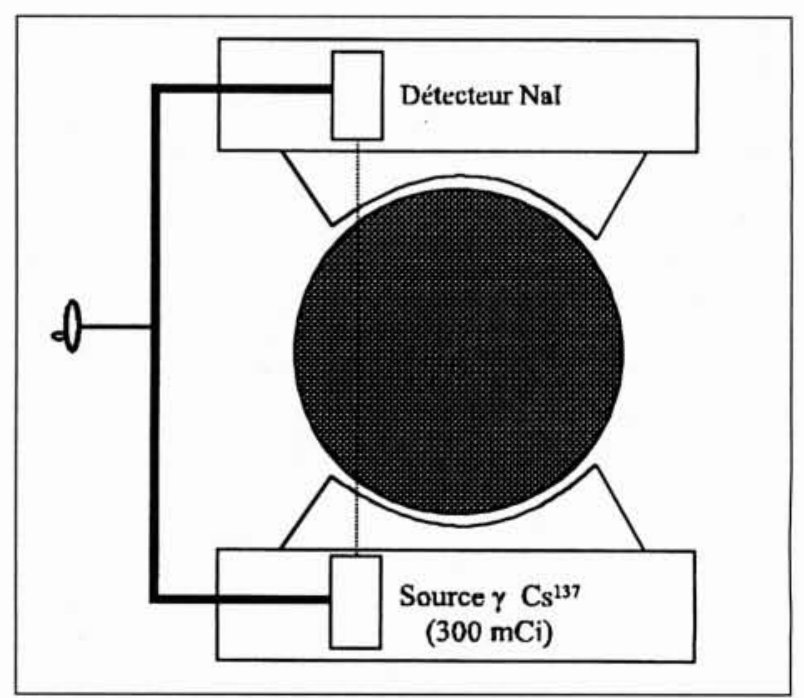

2. Dispositif de mesure tomographique par atténuation de rayons $\gamma$. 


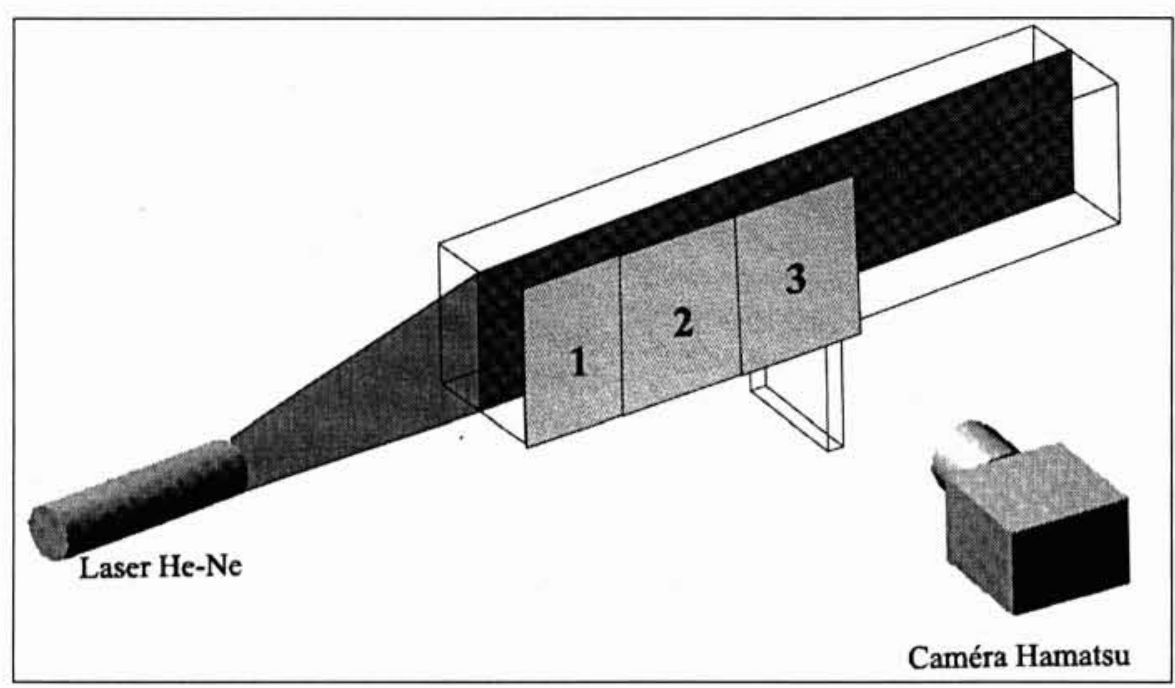

3. Dispositif de mesure des champs de vitesse par PIV.

\subsubsection{Imagerie laser : PIV}

Le deuxième type de mesure globale qui est implémenté concerne la mesure des champs de vitesse suivant une section d'un écoulement en utilisant la technique de Particle Image Velocimetry (PIV). Cette technique a été en particulier utilisée pour déterminer les champs de vitesse dans une entrée de réacteur en écoulement ascendant et en présence de divers internes.

Le principe consiste à éclairer une tranche d'écoulement à l'aide d'une nappe laser. L'écoulement est ensemencé au préalable avec des particules dont la densité est très proche du liquide pour éviter toute vitesse de glissement. La nappe laser obtenue grâce à une lentille cylindrique permet d'éclairer l'écoulement suivant un plan très mince et de focaliser ainsi la prise de vue sur les particules contenues dans ce plan. L'exploitation, par la suite, de deux prises de vue successives permet de déterminer le vecteur déplacement correspondant à chaque particule détectée. Ce calcul est effectué grâce à la technique d'autocorrélation si les deux expositions sont stockées sur la même image ou grâce à la technique d'intercorrélation si les deux expositions sont stockées sur deux images différentes. Le vecteur vitesse correspondant est alors déduit facilement puisque le délai séparant deux prises de vue est connu. La figure 3 présente le dispositif utilisé lors des mesures en entrée de réacteur.

L'acquisition des images est réalisée par une caméra numérique. Trois zones successives ont dû être exposées pour permettre de suivre l'écoulement depuis la conduite d'entrée jusqu'aux parois latérales. La durée de prise de vue varie en fonction des vitesses à mesurer dans chaque zone. Pour une même zone, il peut y avoir localement des différences importantes entre vitesses qui imposent plusieurs séries de prise de vue ayant des temps d'exposition différents.

\section{Limitations}

L'inconvénient de cette technique de mesure réside dans le fait que l'écoulement doit être le plus bi-dimensionnel possible pour que les champs de vitesse mesurés soient représentatifs de l'écoulement. De plus cette technique n'a pour l'instant été testée qu'avec un écoulement monophasique liquide et il serait très intéressant de savoir si elle reste utilisable en présence d'un écoulement diphasique de type écoulement à bulles ou écoulement de gouttelettes dispersées dans du gaz.

\section{- 3.2 Mesures locales}

Compte tenu de la structure très complexe du lit de particules solides, les sondes de mesure de type local restent les seules techniques à l'heure actuelle pour accéder à l'écoulement à travers le lit de catalyseur. En effet la technique de tomographie n'a pas pour l'instant été validée en présence d'écoulement pour un lit de catalyseur réel (billes d'alumine).

\subsubsection{Traçage de la phase liquide}

La méthode du traçage des fluides dans le réacteur permet de détecter des zones mortes ou des zones de passages préférentiels. Utilisée à l'origine de façon globale à travers tout le réacteur, cette méthode est ici mise en œuvre de façon locale afin de détecter des défauts de distribution.

Le principe du traçage consiste à injecter pendant un temps très bref (injection de type impulsion) un volume $V$ de traceur dans l'écoulement à l'amont du réacteur. La concentration en traceur dans l'écoulement est ensuite mesurée en différents points du réacteur. L'analyse comparative des courbes de concentrations obtenues permet de déterminer le temps de séjour moyen correspondant à chaque point de mesure ainsi que le débit local du fluide tracé.

Dans notre cas, le traceur est injecté dans la phase liquide ; il s'agit d'un hydrocarbure dont les caractéristiques physiques, masse volumique et viscosité, sont très proches de celles du fluide étudié. Par exemple, lorsque le fluide étudié est de l'heptane, on utilise de l'hexane comme fluide traceur. La caractéristique physique qui permet de différencier le traceur du fluide porteur est ici l'indice de réfraction. En effet l'heptane a un indice de réfraction proche de 1,39 alors que l'hexane a un indice de réfraction proche de 1,37. Au point de mesure étudié, une sonde de prélèvement permet d'effectuer un échantillonnage en continu du mélange liquide/gaz + traceur. Le gaz est d'abord séparé du mélange liquide/gaz et l'indice de réfraction du mélange liquide/traceur est ensuite mesuré en continu à travers un réfractomètre. On en déduit la courbe de l'écart d'indice de réfraction entre le mélange liquide/traceur et le liquide seul en fonction du temps. Les figures 4 et 5 présentent deux exemples de faisceau de courbes obtenus en plusieurs points de prélèvement du lit de catalyseur d'un réacteur.

L'indice de réfraction du mélange liquide/traceur étant proportionnel à la concentration en traceur, on peut déduire 
4. Exemple de traçage liquide en 4 points de prélèvement d'un même lit de catalyseur.

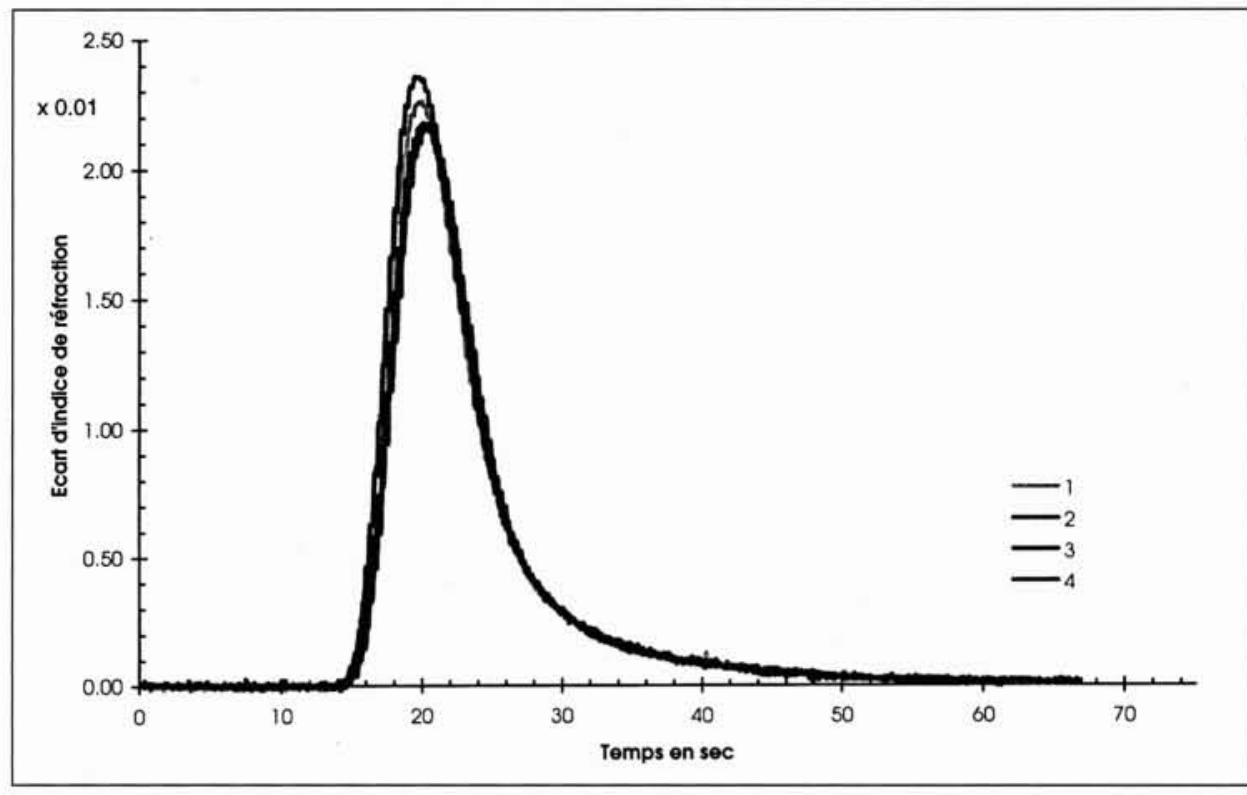

de la figure 4 une très bonne homogénéité de l'écoulement liquide entre les points de mesure. Par contre comme le montre la figure 5 , des défauts de répartition de l'écoulement apparaissent entre les points PA4, PA7 et PA9. De même la comparaison entre les deux niveaux de prélèvement (PA et PC) fait apparaitre un étalement des courbes qui peut être associé à la diffusion de l'écoulement à travers le lit de catalyseur.

\section{Limitations et objectifs}

Sous sa forme actuelle, les problèmes posés par cette méthode sont liés au prélèvement. En effet, il n'est jamais totalement certain que la vitesse de prélèvement soit égale localement à la vitesse de l'écoulement. De même, il peut y avoir une variation de la température entre le point de prélèvement et le point de mesure de l'indice de réfraction. De plus, la mesure en plusieurs points nécessite l'utilisation d'un grand nombre de réfractomètres ou impose de réaliser un essai d'injection par point de mesure.

Aussi, l'utilisation d'un deuxième type d'instrumentation est actuellement à l'étude. Il s'agit d'un système de mesure de l'indice de réfraction par sondes locales dont le volume de mesure est de $1 \mathrm{~mm}^{3}$. Le principe consiste à comparer la réflexion d'une onde de lumière blanche sur deux matériaux différents. Lorsque les deux ondes réfléchies sont en opposition de phase il existe une relation reliant l'indice de réfraction du milieu à la longueur d'onde et à la distance séparant les deux matériaux. Cette technique a évidemment l'avantage de permettre la mesure de l'indice de réfraction in situ de façon peu intrusive. De plus, elle permet la mesure simultanée de l'indice en un grand nombre de points de mesure sans mettre en jeu une batterie de réfractomètres. Cependant à l'heure actuelle, lorsque plusieurs sondes sont utilisées simultanément, des problèmes de dérive optoélectronique
5. Exemple de traçage liquide en 8 points de prélèvements situés à deux hauteurs différentes dans un même lit de catalyseur.

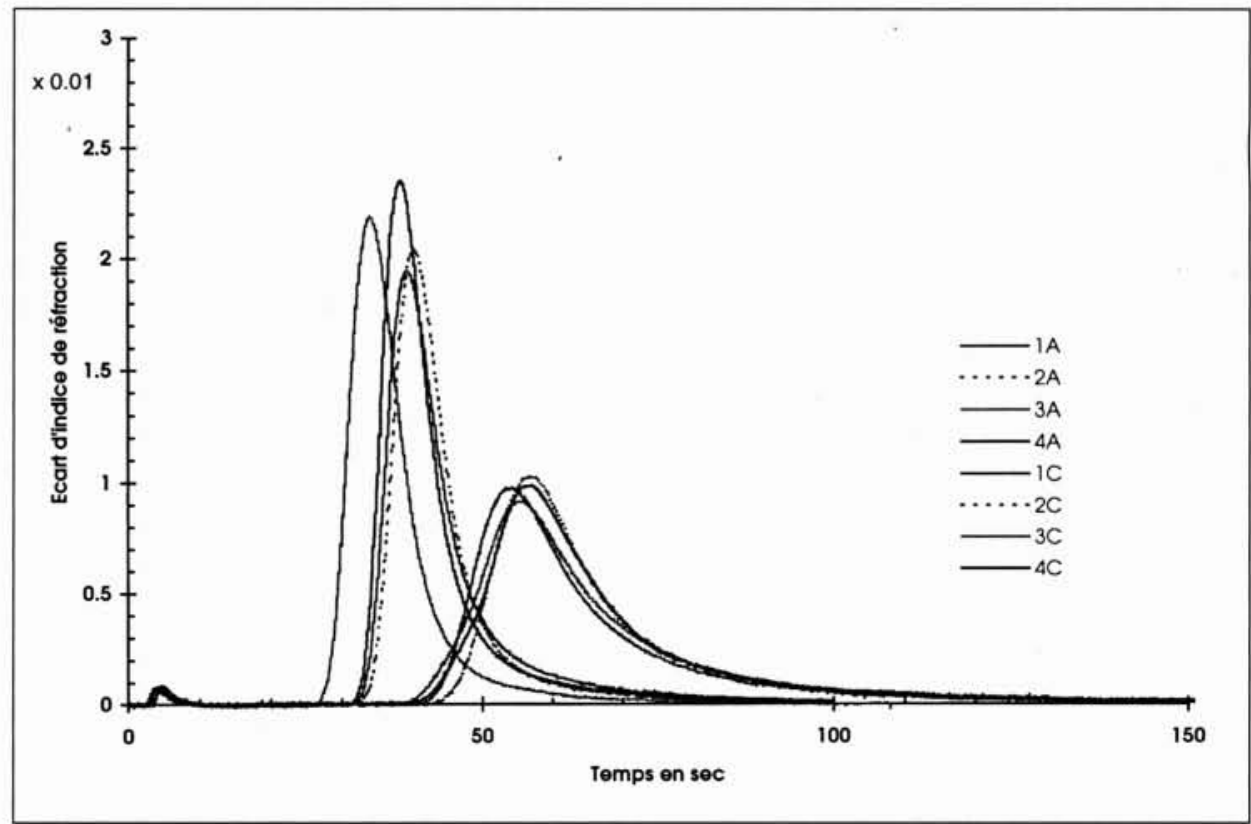


subsistent entre les sondes et obligent une fréquence de recalibration qui est rédhibitoire pour les essais qui sont à effectuer. Un travail de développement est actuellement mené par les constructeurs pour résoudre ces problèmes de dérive.

\subsubsection{Mesure du taux de gaz par sondes optiques}

Les sondes optiques étant adaptées aux mesures locales de taux de vide en présence d'un mélange liquide/gaz, elles ont été tout naturellement retenues pour les mesures à l'intérieur du lit de billes solides de catalyseur. Le principe de mesure bien connu consiste à envoyer un faisceau lumineux dans une fibre optique qui sera soit transmis soit réfléchi par la partie sensible de la sonde suivant que celle-ci est plongée respectivement dans du liquide ou dans du gaz. On obtient donc un signal de type tout ou rien correspondant à la fonction indicatrice de phase dans le mélange.

Dans nos applications, la partie sensible de la sonde optique est en forme de prisme conique en saphir. Le matériau saphir a été choisi pour sa résistance mécanique importante compte tenu de l'environnement de mesure dans lequel la sonde peut être en contact avec les billes de catalyseur. A l'heure actuelle, ces sondes optiques sont utilisées pour effectuer des mesures locales de taux de vide. Les sondes sont déplacées progressivement dans le lit de catalyseur pour déterminer des profils de taux de vide et en déduire des zones caractéristiques de déficit ou d'excès de gaz.

\section{Limitations et objectifs}

Un des problèmes majeurs posés par l'utilisation de sondes optiques au sein d'un lit de billes provient de la détermination d'un seuil séparant le niveau de tension caractéristique du liquide de celui caractéristique du gaz. En effet, comme le montre la figure 6 , le niveau bas du signal, correspondant au liquide est très bruité du fait de réflexions secondaires du signal lumineux sur les grains adjacents à la sonde. Aussi, est-il très délicat de discriminer les signaux dus à ces réflexions parasites de signaux déjà caractéristiques du passage de petites bulles.

Un deuxième problème posé par l'utilisation de ces sondes au sein du lit de billes solides provient de la variation observée de l'amplitude maximale du signal entre deux points de mesure. La position relative de la sonde par rapport aux billes semble influer sur l'amplitude maximale du signal jusqu'à l'annuler lorsque la sonde est en contact avec une bille de catalyseur. Il est donc nécessaire de comprendre si cette atténuation est due à un phénomène caractéristique de l'écoulement (passage de petites bulles) ou est due à l'environnement de la fibre et d'en déduire un critère permettant de décider dans quel cas la mesure reste exploitable.

Parallèlement aux mesures de taux de vide local, une deuxième application de ces fibres optiques est actuellement à l'étude. Il s'agit de mesurer la vitesse et la taille des bulles de gaz. Pour cela deux types de dispositifs vont être testés :

- Mesure de la vitesse des bulles avec une mono-sonde installée face à l'écoulement. On déduit la valeur de la vitesse à partir de la valeur du temps de montée du créneau.

- caractéristique d'une bulle. La fonction reliant temps de montée et vitesse est obtenue à partir d'un étalonnage de la sonde.

- Mesure de la vitesse des bulles avec une bi-sonde installée perpendiculairement à l'écoulement. On détermine le temps de délai moyen entre les deux signaux mesurés par intercorrélation. La vitesse est alors facilement déduite connaissant la distance séparant les deux pointes des sondes.

Une fois la vitesse déterminée, la mesure de la taille de la corde interceptée par la sonde est facilement déduite à partir du temps de passage de la bulle.

Il s'agit de montrer quelle technique est la mieux adaptée pour les mesures de vitesse et de taille de bulles lorsque la fibre est plongée dans le lit de catalyseur.

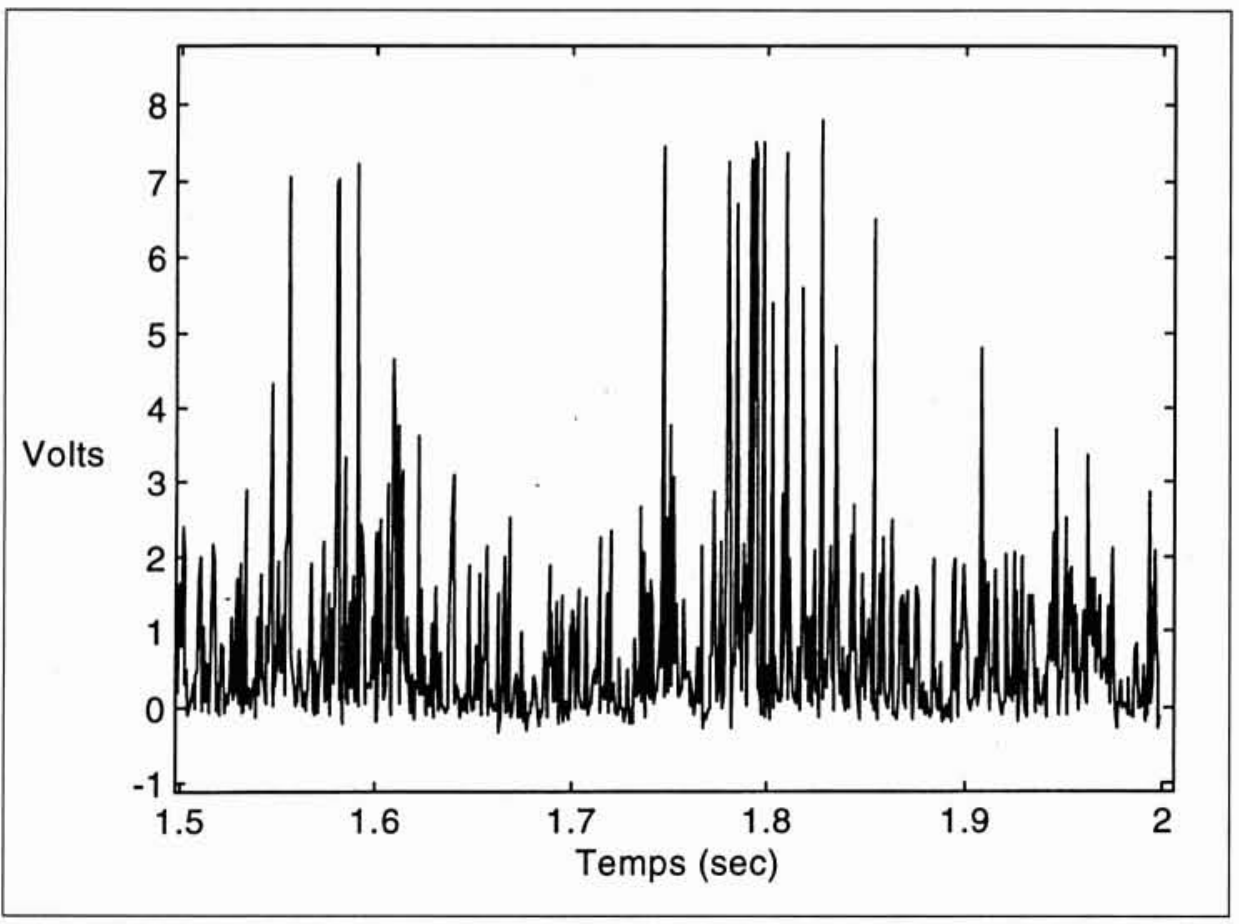

6. Exemple de signal mesuré en présence d'un écoulement liquide/gaz dans le lit de catalyseur. 


\section{IV $\square$ BESOINS}

\subsection{Besoins en instrumentation}

Outre l'amélioration des méthodes décrites dans le paragraphe précédent, il reste à développer et mettre au point des techniques de mesures locales concernant :

- la mesure locale de la vitesse de la phase liquide ou de la phase gaz dans n'importe quelle configuration d'écoulement,

- le traçage de la phase gaz.

\subsection{Besoins en modélisation}

Sur le plan de la modélisation, des besoins apparaissent à deux niveaux :

- Au niveau de l'ensemble de distribution à l'amont du lit. A l'heure actuelle le choix et le dimensionnement des internes permettant d'améliorer la répartition des phases à l'amont du lit se fait à partir de résultats expérimentaux obtenus sur des maquettes. Le dimensionnement d'une unité industrielle réalisé par extrapolation sur la base de règles d'homothétie géométrique pour des vitesses superficielles équivalentes pose parfois problème. Aussi serait-il très intéressant de mettre au point une modélisation bi-dimensionnelle de l'écoulement diphasique au travers des internes de distribution.

- Au niveau du lit. A l'heure actuelle, les tentatives de modélisation des écoulements à travers un lit de particules solides fixes restent limitées. Certains auteurs ont adopté des approches phénoménologiques dans lesquelles ils introduisent des paramètres caractéristiques de la répartition de l'écoulement (facteur de diffusion axiale, radiale, facteur de paroi..) qu'ils déterminent en calant le modèle à des résultats expérimentaux. D'autres auteurs ont proposé un modèle d'écoulement basé sur les équations de bilan de mécanique des fluides qu'ils appliquent à l'écoulement de liquide seul en supposant la fraction du liquide connue expérimentalement en tout point. Un modèle plus complet faisant intervenir les deux phases reste donc à élaborer pour obtenir, pour une porosité du lit donnée, la répartition du taux de gaz et des vitesses suivant une section du lit.

\section{$\mathrm{V} \square \mathrm{CONCLUSIONS}$}

Cet article dresse un bilan des problèmes posés par l'hydrodynamique des réacteurs catalytiques de type gaz/liquide/solide fonctionnant en "lit fixe" et présente l'ensemble de la méthodologie adoptée pour les résoudre. Compte tenu de la complexité des écoulements diphasiques étudiés, l'approche expérimentale est actuellement la voie de recherche privilégiée.

Sur le plan de l'instrumentation, les différentes techniques utilisées sont décrites. L'accent est porté sur les faiblesses de ces techniques pour mesurer correctement un écoulement diphasique à travers un lit de particules solides. De même sont exposés les besoins d'instrumentation complémentaire liés à ces conditions de mesure.

Sur le plan de la modélisation, les besoins restent importants puisqu'aucun modèle d'écoulement diphasique n'est actuellement disponible pour décrire le comportement hydrodynamique de l'écoulement à l'amont du lit ou dans le lit lui-même.

\section{Références}

[1] Kumar S, B., Moslemian D. \& Dudukovic M. P., 1995, A $\gamma$-ray tomographic scanner for imaging viodage distribution in two-phase flow systems, Flow Meas. Instrum., Vol. 6, N) 1, pp.61-73

[2] MacCuaig J. P., Seville J. P. K., Gllbay W. B. \& Clift R., 1985 Application of gamma-ray tomography to gas fluidised beds, Applied Optics, Vol. 24, $\mathrm{N}^{\circ} 23$, pp. 4083-4085.

[3] Toye D., 1997, Etude de l'écoulement ruisselant dans les lits fixes par tomographie à rayons $\mathrm{X}$, Thèse de doctorat, Université de Liège.

[4] Schon J. P., Porcar R., Stanislas M. Royer H. \& Founel T., Les techniques de visualisation qualitatives et quantitatives en mécanique des fluides, Spectra 2000, $\mathrm{N}^{\circ} 164$, pp. 21-26.

[5] Fournel. T., Stanislas M. Arefi S. Hoyez M. C., Chanconie M. AZEMA J. \& BigeARD P., Dépouillement automatique d'images de tomographie laser multi-exposition, 4ème colloque international de visualisation et de traitement d'images en mécanique des fluides, Lille, mai 1990.

[6] CARTELIER A. \& ACHARD J. L., 1991, Local phase detection probes in fluid/fluid two-phase flows, Rev. Sci. Instrum., Vol 61, N², pp. 874-886.

[7] CATELIER A., 1990, Optical probes for local void fraction measurements : charaterization of performance, Rev. Sci. Instrum., Vol 61, N'2, pp. 874-886.

[8] CARTELIER A., 1992, Simultaneous void fraction measurement, bubble velocity and size estimate using a single optical probe in gas-liquid twophase flows, Rev. Sci. Instrum., Vol 63, No11, pp. 5442-5453. 